(2) Der Fettgehalt darf nur durch Entrahmen, Vermischen mit entrahmter Milch oder durch Zusatz von Sahne (Rahm) eingestellt werden.

(1) Wer vorsätzlich oder fahrlässig den Fettgehalt entgegen § I einstellt, begeht eine Zuwiderhandlung im Sinne des $\$ 30$ Abs. 1 Nr. 9 des Milch- und Fettgesetzes.

(2) Verwaltungsbehörde im Sinne des $\$ 36 \mathrm{Abs}$. 2 OWiG ist das Landratsamt, in kreisfreien Städten die Stadtverwaltung.

Diese Verordnung tritt am Tage nach der Verkündung in Kraft.

\title{
Obst und Obsterzeugnisse
}

\section{Bundesrepublik Deutschland}

Zusatz von kolloidaler Kieselsäure zu Tomaten- und Rote-Beete-Pulver

Bekanntmachung des Bundesministeriums für Gesundheitswesen (II B 3-48640-4213 II/68) betr. den Zusatz von kolloidaler Kieselsäure zu Tomaten- und Rote-Beete-Pulver vom 12. September 1968. -

[GMinBl. 19, Nr. 24, S. 347 (1968)]

\section{(Auszug)}

Der Firma Atom GmbH in Karlsruhe, Postfach 21, ist folgende Ausnahmegenehmigung erteilt worden:

Aufgrund des $\$ 20$ a Abs. 2 Nr. 1 des Lebensmittelgesetzes in der Fassung vom 29. Juli 1964 lasse ich im Einvernehmen mit den Herren Bundesministern für Ernährung, Landwirtschaft und Forsten und für Wirtschaft zur Durchführung eines Versuches unter amtlicher Beobachtung ausnahmsweise zu, daß von Ihnen jährlich bis zu $20 \mathrm{t}$ Tomatenpulver und bis zu $3 \mathrm{t}$ Rote-BeetePulver mit einem Zusatz von kolloidaler Kieselsäure hergestellt und in den Verkehr gebracht werden dürfen.

Der Zusatz von kolloidaler Kieselsäure darf höchstens $1 \%$ betragen. Die zugesetzte Kieselsäure muß folgenden Reinheitsanforderungen genügen:

$\begin{array}{lc}\mathrm{SiO}_{2} & 99,9 \% ; \\ \mathrm{Al}_{2} \mathrm{O}_{3} & <0,03 \% \\ \mathrm{TiO}_{2} & <0,06 \% ; \\ \mathrm{Fe}_{2} \mathrm{O}_{3} & <0,003 \% \\ \mathrm{HCl} \text { max } & 0,01 \%\end{array}$

Der Zusatz muß auf den für den Verbraucher bestimmten Packungen, Behältnissen oder Umhüllungen durch die Worte, ,Mit Trennmittel Kieselsäure“" kenntlich gemacht werden. Ferner müssen die Packungen, Behältnisse und Umhüllungen mit einem deutlich sichtbaren Hinweis auf diese Versuchsgenehmigung versehen sein.

Die amtliche Beobachtung des Versuches obliegt der Chemischen Landesuntersuchungsanstalt Karlsruhe, Hoffstr. 3.

Diese Ausnahmegenehmigung gilt bis zum 31.August 1970; sie kann aus wichtigem Grunde vor Ablauf dieser Frist widerrufen werden.

Italien

\section{Alkoholische Genußmittel}

\section{Anerkennung der kontrollierten Ursprungsbezeichnung ,Marsala“6}

Gutachten des Italienischen Nationalkomitees zum Schutz der Ursprungsbezeichnungen der Weine über das Gesuch zur Anerkennung der kontrollierten Ursprungsbezeichnung des Weines, ,Marsala“ und Vorschlag einer entsprechenden gesetzlichen Regelung der Produktion, veröffentlicht am \%. März 1968. [BGesundhbl. 11, Nr. 23, S. 349 (1968)]

Das Nationalkomitee zum Schutz der Ursprungsbezeichnungen der Weine hat den nachstehenden Vorschlag für eine gesetzliche Regelung der Produktion des Dessertweines ,Marsala“ gemacht, zu dem Gesuche und Gegenerklärungen von den interessierten Kreisen innerhalb von 60 Tagen nach der Veröffentlichung an das Ministerium für Landwirtschaft und Forsten gerichtet werden können.

Vorschlag einer gesetzlichen Regelung der Produktion des Dessertweines ,Marsala“

Art. 1: Die kontrollierte Ursprungsbezeichnung, ,Marsala" gilt nur für den Dessertwein, der den Bestimmungen und Anforderungen entspricht, die durch das Gesetz vom 4. November 1950, 